\title{
A micropillar single-photon source design numerically optimized for high efficiency and high indistinguishability
}

Wang, Biying; Denning, Emil Vosmar; Lu, Chao-Yang ; Gregersen, Niels

Published in:

Quantum 2.02020

Publication date:

2020

Document Version

Peer reviewed version

Link back to DTU Orbit

Citation (APA):

Wang, B., Denning, E. V., Lu, C-Y., \& Gregersen, N. (2020). A micropillar single-photon source design numerically optimized for high efficiency and high indistinguishability. In Quantum 2.0 2020 [QTh7B.9] Optical Society of America (OSA).

\section{General rights}

Copyright and moral rights for the publications made accessible in the public portal are retained by the authors and/or other copyright owners and it is a condition of accessing publications that users recognise and abide by the legal requirements associated with these rights.

- Users may download and print one copy of any publication from the public portal for the purpose of private study or research.

- You may not further distribute the material or use it for any profit-making activity or commercial gain

- You may freely distribute the URL identifying the publication in the public portal 


\title{
A micropillar single-photon source design numerically optimized for high efficiency and high indistinguishability
}

\author{
Biying Wang, ${ }^{1,2, *}$ Emil Vosmar Denning, ${ }^{1}$ Chao-Yang Lu, ${ }^{2}$ and Niels Gregersen ${ }^{1}$ \\ ${ }^{1}$ DTU Fotonik, Department of Photonics Engineering, Technical University of Denmark, Building 343, DK-2800 \\ Kongens Lyngby, Denmark \\ ${ }^{2}$ Hefei National Laboratory for Physical Sciences at Microscale, University of Science and Technology of China, \\ Hefei, Anhui, 230026, China \\ *biyiwan@fotonik.dtu.dk
}

\begin{abstract}
We perform numerical optimization of micropillar single-photon source. We present calculations of achievable efficiency and indistinguishability with phonon-induced decoherence, and identify the design parameters providing the best performance. $\odot 2020$ The Author(s)
\end{abstract}

\section{Introduction}

In the past decade, substantial effort within the field of optical quantum information processing has been devoted to the realization of single-photon sources (SPSs) capable of emitting single indistinguishable photons on demand [1]. The SPS figures of merit include the efficiency $\varepsilon$ defined as the number of photons detected by the collection optics per trigger as well as the indistinguishability $\eta$ of the emitted photons. A promising platform for such a source is based on an InAs quantum dot (QD) embedded in semiconductor host material. However, for a QD in a bulk material, the semiconductor-air index contrast limits $\varepsilon$ to a few percent, and additionally, the QD interacts with its solid-state environment leading to phonon-induced decoherence effects limiting the indistinguishability to $\sim 0.8$ even at $0 \mathrm{~K}$ [2]. To control the light emission and ensure a good coupling to the collection optics, it is necessary to place the QD in a structured environment directing the output light towards the microscope objective. In this work, we present a numerical optimization of the micropillar SPS and identify geometrical parameters leading to predicted performance significantly beyond state-of-the-art [1].

\section{Geometry}

The most successful SPS design is the microcavity pillar illustrated in Fig. 1(a). It is a vertical structure consisting of $\lambda$ cavity with a QD in its center surrounded by two distributed Bragg reflectors (DBRs). The light emission is controlled using cavity quantum electrodynamics, where Purcell enhancement is used to enhance the fraction $\beta$ of spontaneous emission coupled to the cavity mode. In addition, the cavity acts to enhance photon emission into the zero-phonon line and suppress emission into the phonon sideband [2] leading to improved indistinguishability without the need for spectral filtering. These assets have led to the demonstrations of highly indistinguishable (>

(a)

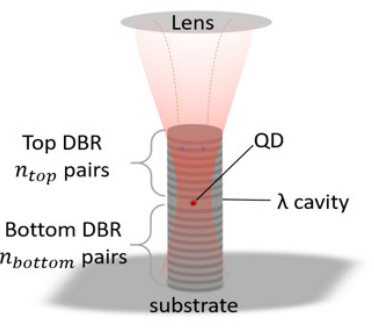

(b)

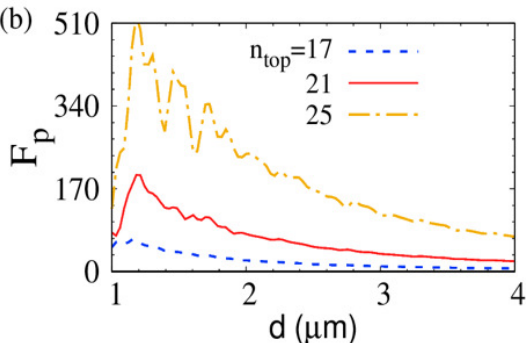

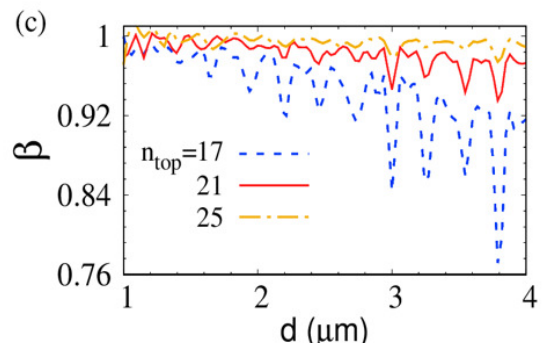

Fig. 1. (a) Illustration of the micropillar geometry. The computed Purcell factor $F_{P}(b)$ and spontaneous emission $\beta$ factor (c) as function of pillar diameter $\mathrm{d}$ and of layer pairs $n_{\text {top }}$ in the top DBR. 
$0.98)$ photons with $\sim 0.6$ extraction efficiency. However, it was recently shown that there is an inherent trade-off between achievable efficiency and indistinguishability for the cavity SPS design [2] in the presence of phononinduced decoherence. Thus an optimization of the micropillar SPS performance requires accurate modeling of the efficiency and the indistinguishability on an equal footing.

In this work, we consider an asymmetric configuration with $n_{\text {bottom }}=40$ layer pairs in the bottom DBR and a varying number $n_{\text {top }}$ of pairs in the top DBR. For each pillar diameter considered, the layer thickness of a DBR layer is chosen as $\lambda_{0} /\left(4 n_{\text {eff }}\right)$, where $\lambda_{0}$ is the design wavelength and $n_{\text {eff }}$ is the diameter-dependent effective index of the fundamental mode.

\section{Results}

We have performed the optical simulations using an open boundary condition Fourier Modal Method [3], while the indistinguishability is computed using a master equation approach in the polaron frame [2].

The computed Purcell factor $F_{p}$ of the micropillar cavity is illustrated in Fig. 1(b) as function of pillar diameter and of top DBR layer pairs $n_{\text {top }}$. For increasing $n_{\text {top }}$, the cavity $Q$ factor is increased leading to an increase in $\mathrm{F}_{\mathrm{p}}$. Additionally, a reduction in the diameter leads to a decrease in the mode volume further enhancing $\mathrm{F}_{\mathrm{p}}$. The increase in $\mathrm{F}_{\mathrm{p}}$ is directly reflected in the calculated spontaneous emission $\beta$ factor presented in Fig. 1(c), which would indicate that the best performance is obtained for the smallest diameter and the largest $n_{\text {top. }}$. However, the total efficiency also depends on the outcoupling to the lens. For small diameters, the output beam is highly divergent, and the predicted collection efficiency presented in Fig. 2(a) is maximized for a diameter above $\sim 2 \mu \mathrm{m}$. The computed indistinguishability shown in Fig. 2(b) initially increases with the Purcell factor as dephasing from phonon is overcome. However, whereas the highest $\varepsilon$ is obtained for $n_{\text {top }}=25, \eta$ drops significantly for this value due to the onset of the strong coupling regime detrimental to the indistinguishability, and the trade-off between high efficiency and high indistinguishability [2] is clearly observed in Figs. 2(a,b). The final figure of merit is the product $\varepsilon \eta$ presented in Fig. 2(c), and we observe that a maximum $\varepsilon \eta$ of $\sim 0.95$ is obtained for $n_{\text {top }}=21$ and a diameter of $2.9 \mu \mathrm{m}$.

In conclusion, we have demonstrated, for the first time, an optimization of micropillar SPS performance by considering efficiency and indistinguishability on an equal footing, and a maximum product $\varepsilon \eta$ of 0.95 is achieved.
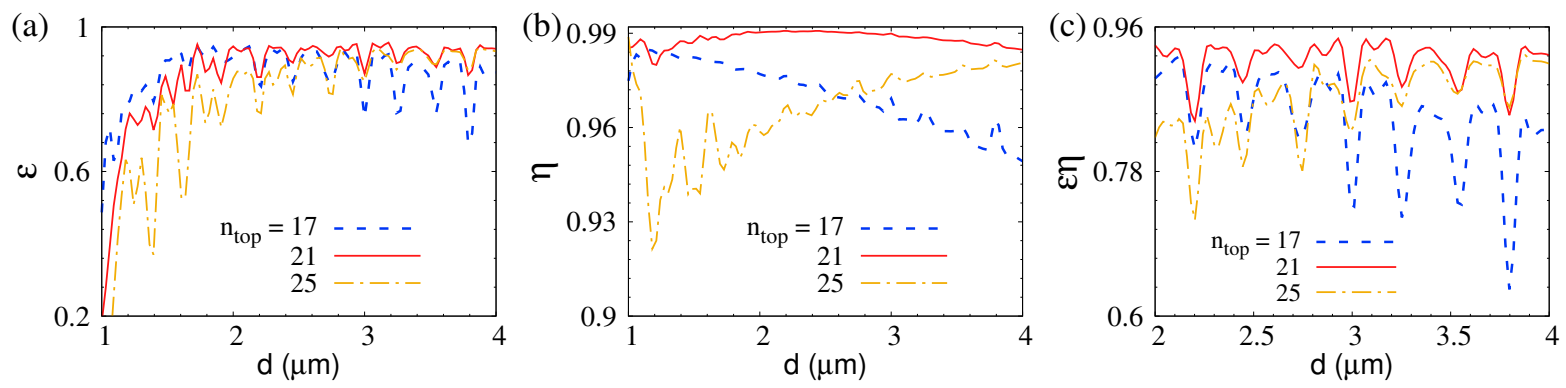

Fig. 2. Computed efficiency $\varepsilon$ (a), indistinguishability $\eta$ (b) and their product $\varepsilon \eta$ (c) as function of pillar diameter $d$ and of layer pairs $n_{\text {top }}$ in the top DBR.

\section{References}

1. X. Ding, Y. He, Z.-C. Duan, N. Gregersen, M.-C. Chen, S. Unsleber, S. Maier, C. Schneider, M. Kamp, S. Höfling, C.-Y. Lu, and J.-W. Pan, "On-Demand Single Photons with High Extraction Efficiency and Near-Unity Indistinguishability from a Resonantly Driven Quantum Dot in a Micropillar", Phys. Rev. Lett. 116, 020401 (2016).

2. J. Iles-Smith, D. P. S. McCutcheon, A. Nazir, and J. Mørk, "Phonon scattering inhibits simultaneous nearunity efficiency and indistinguishability in semiconductor single-photon sources", Nat. Photonics 11, 521526 (2017).

3. T. Häyrynen, J. R. de Lasson, and N. Gregersen, "Open-geometry Fourier modal method: Modeling nanophotonic structures in infinite domains", J. Opt. Soc. Am. A 33, 1298-1306 (2016). 